\title{
26402 - TRIIODO-THYRONINE REVERSES ANGIOTENSIN INDUCED CARDIOMYOCYTE B-MHC EXPRESSION
}

\section{Jingping Ouyang MD; Wuhan University, Wuhan, HUBEI, China}

\section{INTRODUCTION}

Pathological hypertrophy compromises cardiac contractility and could lead the development of heart failure inevitably. Angiotensin II (Ang II) induces an increase in myocardial mass with a compromised contractility accompanied by a shift from the adult isoform alpha myosin heavy chain (á;-MHC, the high ATPase activity isoform) to the fetal isoform a ;-MHC (the low ATPase activity isoform), which is considered as a pathological hypertrophy and inevitably leads to the development of heart failure. In contrast, thyroid hormone-induced cardiac hypertrophy is similar to that observed in physiological hypertrophy which is associated with high cardiac contractility and increased á;-MHC expression. The present study is designed to assess the effect of thyroid hormone on angiotensin II-induced hypertrophic growth of cardiomyocytes in vitro.

\section{METHODS}

Cardiomyocytes were prepared from hearts of neonatal Wistar rats. The effects of Ang II and 3, 3', 5-triiodo-thyronine (T3) alone or their combination (T3 + Ang II) on incorporations of $[3 \mathrm{H}]$-Thymine and $[3 \mathrm{H}]$-Leucine, MHC isoform mRNA expression, protein kinase $\mathrm{C}(\mathrm{PKC})$ activity and $\mathrm{PKC}$ isoform protein expression were studied. RESULTS

Incubation of cultured cardiomyocytes with Ang II resulted in enhanced [3H]-Leucine incorporation, â;-MHC mRNA expression, PKC activity and PKCå; expression, and reduced á;-MHC mRNA expression as compared to cultured Cardiomyocytes treated with culture medium alone $(\mathrm{P}<0.05)$. T3 treatment prevented Ang II induced increases in PKC activity, PKCå; and â;-MHC mRNA overexpression and favored á;-MHC mRNA expression $(\mathrm{P}<0.05, \mathrm{~T} 3+$ Ang II vs Ang II).

\section{CONCLUSION}

Thyroid hormone appears to be able to reprogram gene expression in Ang II-induced cardiac hypertrophy and PKC signal pathway may be involved in such remodeling process. 